\title{
Gauging contact line friction of droplets: In situ measurement within a digital microsystem
}

\author{
Johannes Theisen ${ }^{\mathrm{a}}$, Laurent Davoust ${ }^{\mathrm{b}, *}$ \\ ${ }^{a}$ University of Grenoble (UJF), Laboratory of Geophysical and Industrial Fluid Flows (LEGI), Domaine universitaire, 38400 Saint Martin d'Hères, France

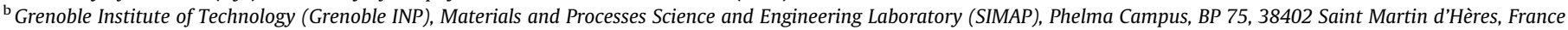

Here is presented an interferometry technique to quantify damping of electrowetting induced shape oscillations of a sessile microdrop. This characterization method and its devoted chip can be inserted into a microsystem to quantify contact line friction and hence surface ageing in digital electrowetting applications (lab-on-chips, microfluidic lenses and displays). Here, the contact line friction is proposed as a criteria to distinguish sensor surfaces contaminated by adsorbed bio-molecules.

\section{Introduction}

Microsystems using droplet microfluidics are subject to a major challenge: how to circumvent contact line friction, which opposes itself to drop motion, and, obviously, how to characterize it? The aim of this paper is to deliver a surface degradation analysis based on wetting abilities, which can be inserted into micro-systems such as lab-on-a-chips [1], microfluidic optical lenses [2] or display technologies [3]. The characterization of dynamical wetting is of prior importance for quantifying the response time and stabilization of droplet motions involved in these systems. Here, the contact line friction is proposed as a sensitive criteria to distinguish between degraded and non-degraded surfaces.

The transduction mechanism used for quantifying the contact line friction is the transient regime of decaying, sessile droplet shape oscillations, initially induced by electrowetting on dielectrics (EWOD). EWOD is now a technology commonly used in digital lab-on-a-chips, for drop motion, mixing enhancement, break-up/ coalescence of drops, aliquoting and other basic manipulations. EWOD is used as well in meniscus-based lenses to control the focal length and in display technologies to create a luminosity contrast, for instance. The capillary wave damping results mainly from the contact line friction on the substrate, especially when the size of the drop is increasingly smaller. The friction is composed of a linear friction term, proportional to the velocity of the contact line, and a non-linear term accounting for stick-slip motion due to surface

\footnotetext{
* Corresponding author.

E-mail address: laurent.davoust@simap.grenoble-inp.fr (L. Davoust).
}

roughness. Viscous damping in the drop volume can be neglected for water, as the corresponding time scale is much greater than the time scale for contact line friction $[4,5]$.

Here is presented an interferometry technique to measure damping of shape oscillations of a sessile microdrop induced by coplanar electrowetting, without the need for any thin wire serving as counter-electrode. The technique consists in measuring the interface displacement of the drop apex relative to the substrate by interferometry. The interferometry signal, which is frequencymodulated by the velocity of the interface, is related to the amplitude of the drop oscillation. This point is of prior importance: even small amplitudes, down to the order of several times the laser wavelength of $532 \mathrm{~nm}$, may be detected with a nice precision. One full period of the interferometry signal corresponds to the displacement of the interface over an optical path of one wavelength.

The novelty of this approach is the combination of a facile in situ analysis of droplet shape oscillations, with a fast and highly accurate measurement by laser interferometry, even for small amplitudes $<10 \mu \mathrm{m}$, which is not possible with standard imaging techniques. The easy integration of this technology in microfluidic systems based on electrowetting in a coplanar, non-intrusive electrode configuration is an inherent feature of the developments presented in this paper.

\section{EWOD as an actuation mechanism}

The electrowetting setup is realized with coplanar electrodes [6-8], which has two major advantages over the two intrusive EWOD configurations with diving [9] or catenary [10] 
counter-electrode: first of all, a facile interferometry measurement is impossible in these configurations, secondly, the presence of a wire-induced meniscus at the drop apex and the subsequent viscous friction due to the supplementary contact line would strongly modify the drop dynamics.

The geometry of the coplanar electrowetting electrodes consists of two half-moons of base width $2 \mathrm{~mm}$ facing each other with a gap of $3 \mu \mathrm{m}$, as shown in Fig. 1. The electrodes are covered by two coatings, an insulating layer $\left(\mathrm{Si}_{3} \mathrm{~N}_{4}\right.$, silicon nitride, thickness $600 \mathrm{~nm}$ ) and a hydrophobic layer (SIOC silicon carbide, thickness $1000 \mathrm{~nm}$ ). This technology has been demonstrated to exhibit high electrowetting ability as well as high resistance against dielectric break-down, which is a prior feature as far as coplanar electrode designs are concerned.

The electrical potential applied to the electrode pair is composed of two AC voltages $U_{1}$ (frequency $v$ ) and $U_{2}$ (frequency $f_{\text {exc }}$ ), provided by two frequency generators. These two signals are processed by an analog multiplier furnishing a voltage $U_{3}=\left(1+U_{2}\right)$ $10 \mathrm{~V}) U_{1}$. This resulting voltage $U_{3}$ is amplified by a high-voltage amplifier delivering the input actuation $U=100 U_{3}$. The electrode pair has one reference electrode connected to the mass, while the second coplanar electrode is connected to the voltage $U$.

The decisive advantage in making use of dual-frequency electrowetting is that, when imposing a high frequency $v=20 \mathrm{kHz}$ to $U_{1}$, the amplitude of $U_{1}$ may be used to tune the contact angle (for instance $\theta=\pi / 2$ ) in a range, which depends on the composition of the underlying substrate and contact angle hysteresis (see also [11] for another application of dual-frequency electrowetting). Due to the minimization of electrostatic energy, the voltage contribution $U_{1}$ makes the droplet straddle the electrodes [7], such that its position is much more stable than in usual geometries. Therein lies a second feature of prior importance, since interferometry measurements on a curved surface need high local precision to be feasible. The voltage modulation $U_{2}$, characterized by a low frequency in the range of $f_{\text {exc }} \sim 10-1000 \mathrm{~Hz}$, is used to displace radially inwards and outwards the contact line inducing drop shape oscillations of frequency $f=2 f_{\text {exc }}$, thereby controlling perfectly a spherical wave network along the surface of the droplet.

The drops with an initial volume of $1.5 \mu \mathrm{L}$ consist of ultra-pure water (resistivity $18.2 \mathrm{M} \Omega$ ). The surface tension is measured to be $57 \mathrm{mN} / \mathrm{m}$ with the Wilhelmy technique using a NIMA tensiometer.

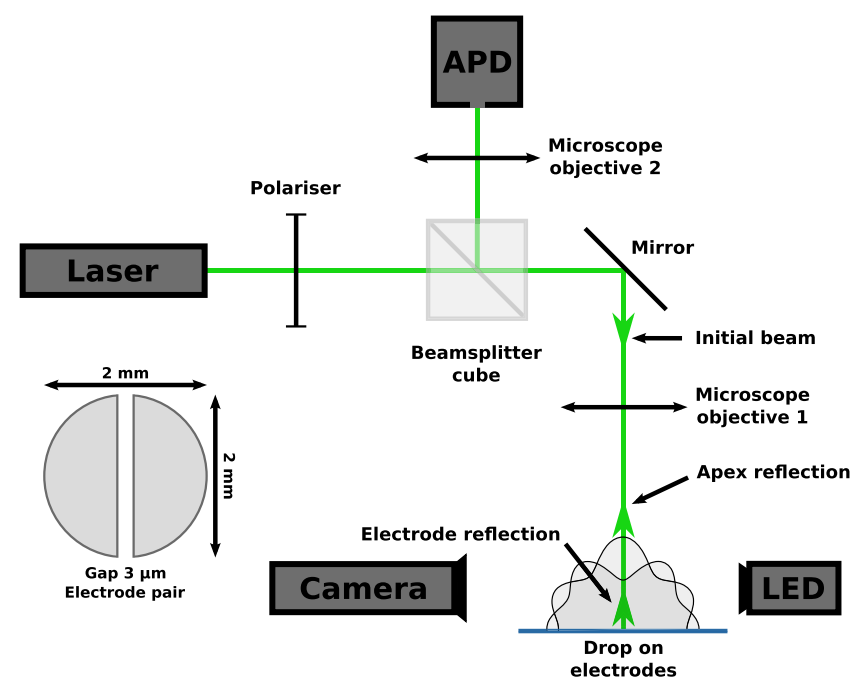

Fig. 1. Setup of the interferometry system. The laser beam traverses the optical system to form two reflections on the drop apex and the electrode, which are detected by the avalanche photo diode (APD). Insert: Electrode pair in use for electrowetting actuation.
Due to residual traces of contamination, the surface tension is always lower than expected from theory. The drops are deposited on the electrodes, which are covered by a very thin film of silicone oil (viscosity $6 \mathrm{mPa}$ s) to avoid stick-slip motion and a too large value of the contact angle hysteresis as well. The silicone oil is used in six different solutions of $0 \mathrm{mM}, 0.25,1,2.5,3.75$ and $5 \mathrm{mM}$ palmitic acid (PA). The PA is added to the silicone oil from a $1 \mathrm{mM}$ solution of chloroform and PA.

\section{Optical measurement of contact line friction}

A dedicated Michelson interferometer is developed to detect the vertical motion of the apex of a sessile drop: a laser beam (wavelength $\mathrm{L}=532 \mathrm{~nm}$ ) horizontally crosses a polarizer, a beam splitter and is directed onto a mirror, where it is reflected vertically onto the drop's apex after being focused by an inverted microscope objective, located above the drop (cf. Fig. 1). The drop surface gives rise to a first reflection. A second reflection is generated at the solid substrate, on which the drop rests. These two reflections take the same way back to the beam splitter, where they are redirected through a second inverted objective for focusing the laser beam and collecting the maximum light intensity on the active surface of an avalanche photo diode at the focal point.

The delivered light intensity $I$ at the avalanche photo diode (APD) follows the law[12]:

$I \propto I_{\mathrm{s}}+I_{\mathrm{a}}+2 \sqrt{I_{\mathrm{s}} I_{\mathrm{a}}} \cos \left(\frac{4 \pi}{L}\left[(\widetilde{n}-1) \varsigma(t)+\left(\widetilde{n}_{R}-\varsigma_{o}(f)\right)\right]\right)$,

where $\widetilde{n}$ is the refractive index of the liquid, $R$ is the instantaneous drop radius, $I_{\mathrm{s}}$ and $I_{\mathrm{a}}$ are the light intensities received from the substrate and the drop apex, respectively. The oscillation amplitude of the vertically moving apex is $\zeta(t)=\zeta_{0}(f) \sin (2 \pi f t)$.

The signal detected at the APD is delivered to an analog filter (Krohn-Hite model 3362, band-pass $1 \mathrm{kHz} / 200 \mathrm{kHz}$, type Butterworth) prior to recording at a sampling frequency of $250 \mathrm{kHz}$. Before each measurement, a delay of $0.3 \mathrm{~s}$ is systematically imposed to the system in order to reach the steady regime of the droplet's oscillations. This is the initial condition of the experiments conducted in this paper. At the initial time, the forcing is switched off by removing the voltage $U_{2}$ and the drop oscillation quickly switches to its natural frequency while being damped under contact line friction [13]. The APD signal is then recorded for $150 \mathrm{~ms}$. An example of the steady signal prior to cutting off is given in Fig. 2 for one period of oscillation. The excitation frequency $f_{\text {exc }}$ is chosen near the natural frequency [9]:

$f_{2}=\sqrt{\frac{2 C \gamma}{\pi^{2} \rho R^{3}}}=\sqrt{\frac{4 C \gamma}{3 \pi \rho V}}=247 \mathrm{~Hz}$,

where $C=3.77$ is a correction factor for the natural frequency of a sessile drop with contact angle $\pi / 2$ [14], $\gamma$ and $\rho$ are the surface tension and the density of water and $V$ the drop volume $(V=10 \mu L)$.

As already mentioned, the apex of the droplet is used as a semireflecting mirror for interferometry to characterize the oscillation amplitude with two main advantages: first, the amplitude of the oscillating droplet is the largest at its apex, where the oscillatory displacement is perfectly vertical and standing (antinode). Second, the area in the vicinity of the apex remains parallel to the underlying solid substrate whilst translating vertically to it. With a precise optical alignment, which means a laser beam impacting at a right angle onto the electrodes, one obtains a reproducible and easy to process interferometry signal (cf. Fig. 2).

The evaluation of the interferometry signal is based on an algorithm of frequency detection. In order to correctly evaluate the amplitude of the signal (cf. Fig. 3), the phase shift is calculated and only the data of fully available periods of the signal are considered. 


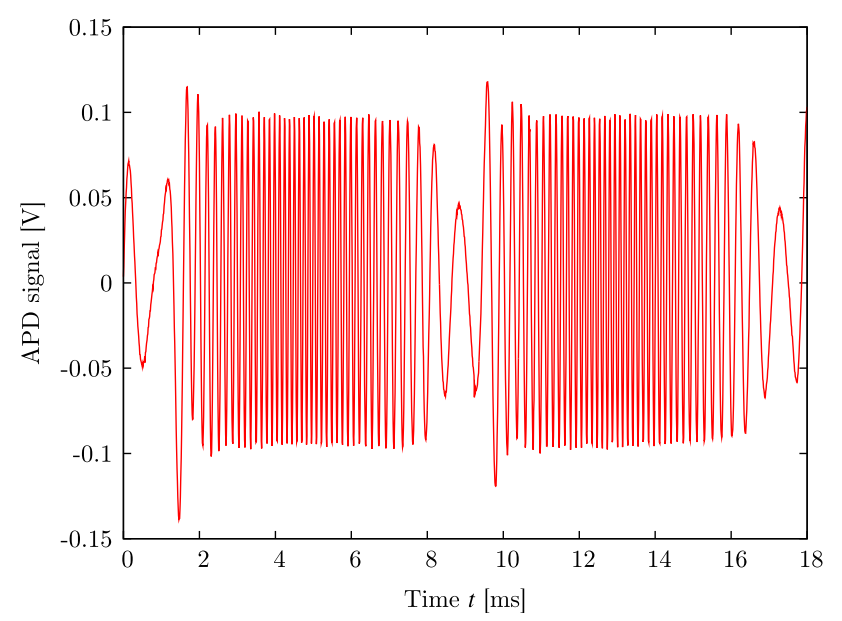

Fig. 2. Interferometry signal of one period of oscillation of a $10 \mu \mathrm{L}$ drop at $f=60 \mathrm{~Hz}$ oscillation frequency.

For each of these periods, the local extrema due to sweepings of the optical path are counted and weighted by the absolute value of the voltage amplitude, $\left|U_{\mathrm{APD}}\right|$, delivered by the APD:

$\zeta_{0}^{j} \propto \sum_{i=1}^{N}\left|U_{\mathrm{APD}}\right| \delta_{t, t^{i}}$,

where $\widetilde{n}$ is the total number of local extrema contained in one halfperiod of oscillation, which corresponds to one burst, $\zeta_{0}^{j}$ is the oscillation amplitude calculated from one given burst $j, i$ designates the $i$ th extremum during this burst, $\delta$ is the Kronecker symbol, $t$ is the time and $t^{i}$ is the time corresponding to the $i$ th extremum. The time-dependent amplitude is then fitted to an exponential curve, whose decay rate is used for identification of the friction coefficient. The data used for the fit ranges between an initial data point with amplitude $\zeta_{0}^{1}$, and a final data point for which the amplitude is no less than $0.1 \zeta_{0}^{1}$. This threshold on the measured amplitude is chosen so as to exploit bursts with a high enough number of fringes.

\section{Under-damped droplet shape oscillations}

The foundation of droplet shape oscillations has been laid by Lamb [15], and more recently, by Kang [9] for hemi-spherical drops. Studies on the decay regime of capillary waves on droplets have been published by Kang [16] and Garimella [4] for overdamped systems. Kang gives an expression for contact line friction in their model of free drop oscillations:

$$
\begin{aligned}
\ddot{\zeta}_{n} & +2(n-1)(2 n+1) \frac{\mu}{\rho R^{2}} \dot{\zeta}_{n}+\frac{2 n \lambda}{\rho R^{2}} A_{n n} \dot{\zeta}_{n}+n(n-1)(n-2) \frac{\gamma}{\rho R^{3}} \zeta_{n} \\
& =-\frac{2 n \lambda}{\rho R^{2}} \sum_{k=2, k \neq n}^{\infty} A_{n k} \dot{\zeta}_{k} .
\end{aligned}
$$

Here, stick-slip motion of the contact line is neglected, since the substrate is lubricated. The variables $\zeta_{n}, \dot{\zeta}_{n}$ and $\ddot{\zeta}_{n}$ correspond to the amplitude, velocity and acceleration of the oscillation mode $n$, respectively. The second term on the left hand side takes into account the viscous damping with viscosity $\mu$ in the volume. The third term is a linear friction term with a friction coefficient $\lambda$. The weighting factor in the fourth term, proportional to $\zeta_{n}$, is the square of the resonance frequency for mode $n$. The right hand side of (2) takes into account the non-linear coupling of the oscillation modes $k \neq n$ due to contact line friction. In Kang's work [16], this nonlinear coupling is of importance, as they work with a step in the applied voltage, exciting all of the oscillation modes during the over-damped transition. In this work, dealing with underdamped oscillations, one may neglect the self-sustained coupling, as the oscillations rapidly adopt the natural frequency of our system, namely $f_{2}$ [13]. As a result, the oscillation mode 2 (oblate-prolate) is the preponderant oscillation mode after the actuation has been switched off. In this case, Eq. (2) may be simplified to:

$\left.\ddot{\zeta}_{2}+25 \frac{\mu}{\rho R^{2}}+\frac{2 \lambda}{\rho R^{2}}\right) \dot{\zeta}_{2}+8 \frac{\gamma}{\rho R^{3}} \zeta_{2}=0$.

Using basic theoretical ingredients of harmonic oscillator theory, the damping time scale $\tau$ may be derived as:

$\frac{1}{\tau}=(5 \mu+2 \lambda) \frac{1}{\rho R^{2}}$.

From this expression follows the contact line friction coefficient:

$\left.\lambda=\frac{1}{2} \frac{\rho R^{2}}{\tau}-5 \mu\right)$.

\section{Results}

The amplitude attenuation for a $1.5 \mu \mathrm{L}$ water droplet with an EWOD-induced contact angle, $\pi / 2$, on a SiOC substrate covered by a thin drained silicone oil film (viscosity: $6 \mathrm{mPa}$, molar concentration in palmitic acid: $0 \mathrm{mM}$ ) is shown in Fig. 4. The exponential fit gives a damping factor of $22.88 \mathrm{~s}^{-1}$, or respectively an attenuation time constant of $\tau=43.71 \mathrm{~ms}$, corresponding to a contact line friction of $\lambda=0.013 \mathrm{~N} \mathrm{~s} / \mathrm{m}^{2}$. In comparison with the experimental data of Garimella [4] and Kang [16], who find 0.4 and $0.18 \mathrm{~N} \mathrm{~s} / \mathrm{m}^{2}$, respectively for non-lubricated Teflon substrates, this value seems reasonable. Fig. 5 shows the results for the different concentrations of palmitic acid in the silicone oil film. With increasing concentration of PA, the contact line friction coefficient increases.

\section{Final remarks}

In this paper, interferometry is demonstrated to be a very convenient detection method to measure the under-damped decay of surface capillary waves on sessile drops, induced by electrowetting.

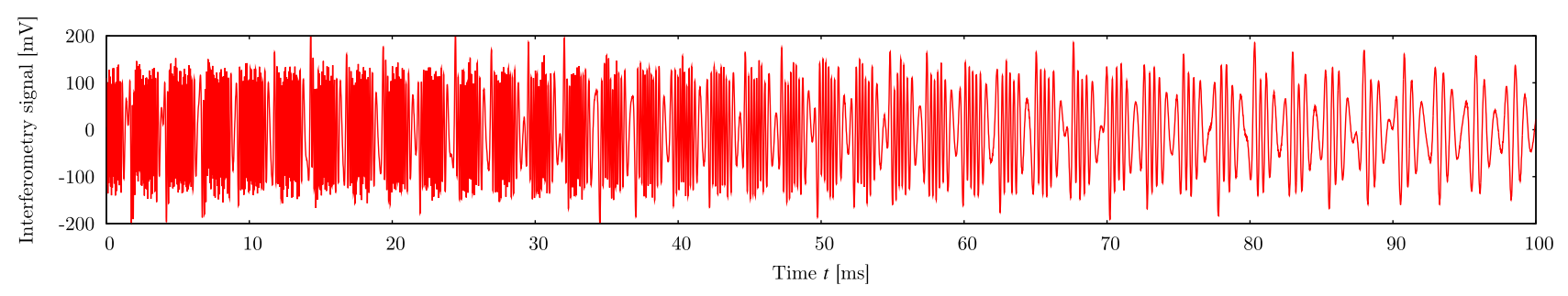

Fig. 3. Interferometry signal of decaying droplet shape oscillations. With diminishing oscillation amplitude, the burst contains less extrema. 


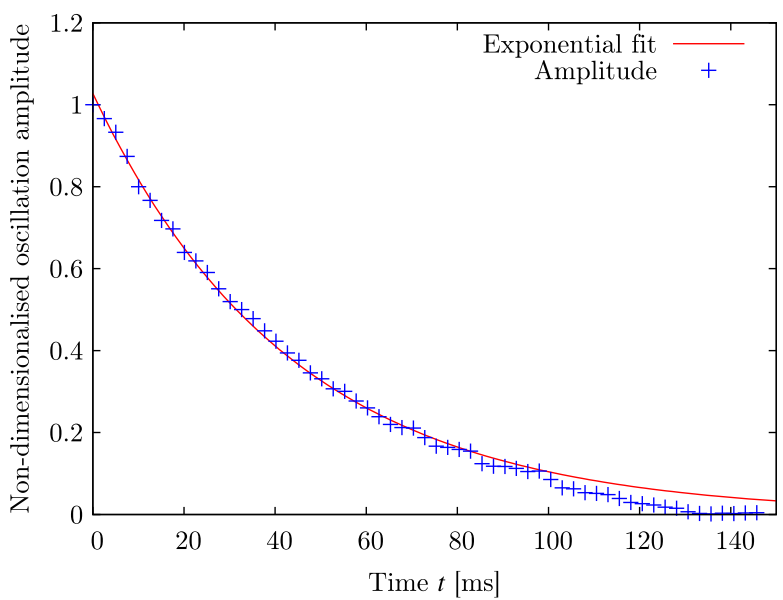

Fig. 4. Amplitude attenuation measured by interferometry. The line corresponds to a fit with a damping time of $\tau=43.71 \mathrm{~ms}$.

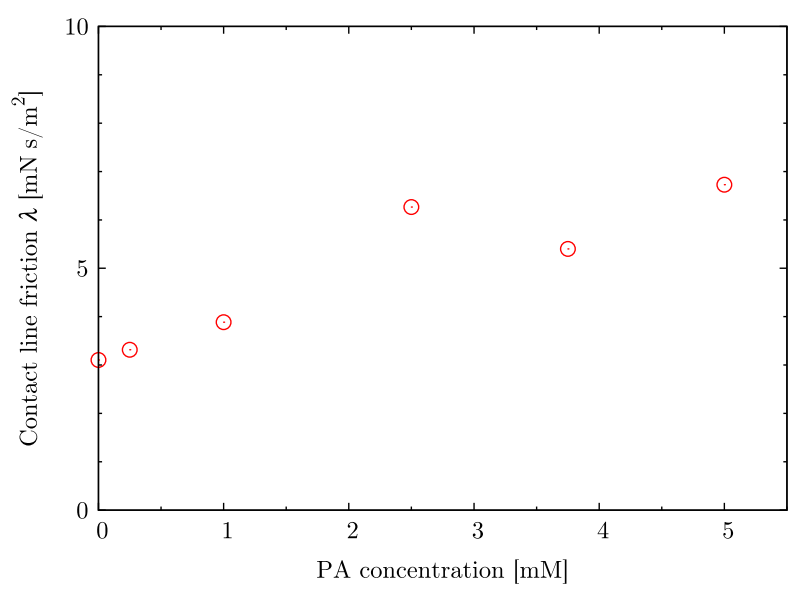

Fig. 5. Contact line friction coefficient $\lambda$ for different concentrations of palmitic acid in the silicone oil film.
The time-dependent amplitude of the moving drop apex is shown to precisely translate the exponential attenuation of the oscillations, as illustrated here for a lubricated SiOC substrate. By contaminating the lubricating silicone oil with fatty acids, surface degradation is translated in an increase of the friction coefficient. As a means of drop shape oscillation detection, interferometry performed at the drop apex exhibits several advantages. The measurement of apex motion in the order of one laser wave length is much more precise as image analysis and permits the analysis of small amplitude interface motions. In addition, the technique is adapted to situations, in which imaging could not be conveniently performed, as is the case for most microfluidic applications in micro systems. For instance, the proposed methodology can be performed in confined geometries such as digital lab-on-a-chips and liquid lenses, thus providing design guidelines as well as a criteria to characterize the surface degradation of EWOD chips.

\section{References}

[1] M.G. Pollack, A.D. Shenderov, R.B. Fair, Lab Chip 2 (2002) 96-101.

[2] B. Berge, J. Peseux, Eur. Phys. J. E 3 (2000) 159-163.

[3] R.A. Hayes, B.J. Feenstra, Nature 425 (2003) 383-385

[4] S.R. Annapragada, S. Dash, S.V. Garimella, J.Y. Murthy, Langmuir 27 (13) (2011) 8198-8204

[5] T. Funada, D. Joseph, J. Wang, Potential Flows of Viscous and Viscoelastic Fluids, Cambridge University Press, New York, 2008.

[6] U.-C. Yi, C.-J. Kim, J. Micromech. Microeng. 16 (10) (2006) 2053-2059.

[7] P. Sen, C.-J. Kim, Langmuir 25 (8) (2009) 4302-4305.

[8] R. Malk, A. Rival, Y. Fouillet, L. Davoust, ASME Conference Proceedings 2010 (54501) (2010) 239-248.

[9] K.H. Kang, J.M. Oh, S.H. Ko, Langmuir 23 (15) (2008) 8379-8386.

[10] P. Dubois, G. Marchand, Y. Fouillet, J. Berthier, T. Douki, F. Hassine, S. Gmouh, M. Vaultier, Anal. Chem. 78 (2006) 4909-4917.

[11] J. Theisen, L. Davoust, Langmuir 28 (1) (2012) 1041-1048.

[12] L. Davoust, J.L. Achard, A. Cartellier, Prog. Coll. Pol. Sci. 115 (2000) 249-254

[13] D.M. Henderson, K. Larson, Y.K. Rao, Langmuir 7 (1991) 2731-2736.

[14] M. Strani, F. Sabetta, J. Fluid Mech. 141 (1984) 233-247.

[15] H. Lamb, Hydrodynamics, Cambridge University Press, 1932.

[16] K.H. Kang, J.M. Oh, S.H. Ko, Phys. Fluids 22 (2010) 032002. 